\title{
Higher socioeconomic status is related to healthier levels of fatness and fitness already at 3 to 5 years of age: The PREFIT project
}

Ignacio Merino-De Haro, Jose Mora-Gonzalez, Cristina Cadenas-Sanchez, Pere A. Borras, Pedro J. Benito, Oscar Chiva-Bartoll, Coral Torrijos-Niño, Cristina Samaniego-Sánchez, José Javier Quesada-Granados, Alejandro Sánchez-Delgado, Cecilia Dorado-García, José M. García-Martínez, Germán Vicente-Rodríguez, Idoia Labayen, Francisco B. Ortega \& on behalf of the PREFIT project group

To cite this article: Ignacio Merino-De Haro, Jose Mora-Gonzalez, Cristina Cadenas-Sanchez, Pere A. Borras, Pedro J. Benito, Oscar Chiva-Bartoll, Coral Torrijos-Niño, Cristina SamaniegoSánchez, José Javier Quesada-Granados, Alejandro Sánchez-Delgado, Cecilia Dorado-García, José M. García-Martínez, Germán Vicente-Rodríguez, Idoia Labayen, Francisco B. Ortega \& on behalf of the PREFIT project group (2018): Higher socioeconomic status is related to healthier levels of fatness and fitness already at 3 to 5 years of age: The PREFIT project, Journal of Sports Sciences, DOI: 10.1080/02640414.2018.1558509

To link to this article: https://doi.org/10.1080/02640414.2018.1558509

View supplementary material $\llbracket$ Published online: 27 Dec 2018.

Submit your article to this journal $๘$

山 Article views: 19

View Crossmark data $[\pi$ 


\title{
Relation between socioeconomic status, fatness and fitness in preschoolers
}

\author{
Ignacio Merino-De Haro*a, Jose Mora-Gonzalez ${ }^{* a}$, Cristina Cadenas-Sanchez ${ }^{a}$, Pere A. Borras ${ }^{b}$, Pedro J. Benitoc, \\ Oscar Chiva-Bartoll ${ }^{d}$, Coral Torrijos-Niño ${ }^{e}$, Cristina Samaniego-Sánchez ${ }^{\dagger}$, José Javier Quesada-Granados ${ }^{f}$, \\ Alejandro Sánchez-Delgado (10) ${ }^{9}$, Cecilia Dorado-Garcíah , José M. García-Martínez ${ }^{i, j}$, Germán Vicente-Rodríguez (10), \\ Idoia Labayen', Francisco B. Ortega ${ }^{\mathrm{a}, \mathrm{m}}$ and on behalf of the PREFIT project group ${ }^{* *}$
}

\begin{abstract}
aPROFITH "PROmoting FITness and Health through physical activity" Research Group, Department of Physical and Sports Education, Faculty of Sports Science, University of Granada, Granada, Spain; ${ }^{b}$ Physical activity and sport sciences research group, Schools for Health Europe Network Research Group, University of the Balearic Islands, Palma, Spain; 'LFE Research Group, Department of Health and Human Performance, Faculty of Physical Activity and Sport Science-INEF, Universidad Politécnica de Madrid, Madrid, Spain; ${ }^{\circ}$ Department of Education and Specific Didactics, LIFE Research Group, University of Jaume I, Castellón, Spain; eHealth and Social Research Center, University of Castilla-La Mancha, Cuenca, Spain; fDepartment of Nutrition and Food Sciences, Faculty of Pharmacy, University of Granada, Granada, Spain; ${ }^{9}$ Department of Physical Education, Faculty of Education Sciences, University of Cádiz, Puerto Real, Spain; ${ }^{h}$ Research Institute of Biomedical and Health Sciences (IUIBS), Dept Physical Education, Palmas de Gran Canaria University, Las Palmas, Spain; 'Department of Education, Faculty of Education Sciences, University of Almeria, Almería, Spain; jSPORT Research Group (CTS-1024), CERNEP Research Center, University of Almería, Almería, Spain; ${ }^{k}$ GENUD (Growth, Exercise, NUtrition and Development) research group, Instituto Agroalimentario de Aragón (IA2), Centro de Investigación Biomédica en Red de Fisiopatología de la Obesidad y Nutrición (CIBERObn), Faculty of Health and Sport Science (FCSD, Ronda Misericordia 5, 22001-Huesca, Spain), Department of Physiatry and Nursing, University of Zaragoza, Zaragoza, Spain; 'Institute for Innovation \& Sustainable Development in Food Chain (IS-FOOD), Public University of Navarra, Campus de Arrosadía, Pamplona, Spain; mDepartment of Biosciences and Nutrition, Karolinska Institutet, Huddinge, Sweden
\end{abstract}

\begin{abstract}
This study aimed to analyse the association between socioeconomic status (SES) and fatness and fitness in preschoolers. 2,638 preschoolers (3-5 years old; $47.2 \%$ girls) participated. SES was estimated from the parental educational and occupational levels, and the marital status. Fatness was assessed by body mass index (BMI), waist circumference (WC), and waist-to-height ratio (WHtR). Physical fitness components were assessed using the PREFIT battery. Preschoolers whose parents had higher educational levels had lower fatness $(P<0.05)$. BMI significantly differed across occupational levels of each parent $(P<0.05)$ and WHtR across paternal levels $(P=0.004)$. Musculoskeletal fitness was different across any SES factor $(P<0.05)$, except handgrip across paternal occupational levels $(P \geq 0.05)$. Preschoolers with high paternal occupation had higher speed/agility $(P=0.005)$, and those with high or low maternal education had higher $\mathrm{VO}_{2} \max (P=0.046)$. Odds of being obese and having low musculoskeletal fitness was lower as SES was higher $(P<0.05)$. Those with married parents had higher cardiorespiratory fitness than single-parent ones $(P=0.010)$. School-based interventions should be aware of that children with low SES are at a higher risk of obesity and low fitness already in the first years of life.
\end{abstract}

ARTICLE HISTORY Accepted 6 December 2018

\section{KEYWORDS}

Body composition; physical fitness; preschoolers; socioeconomic factors

\section{Introduction}

Childhood obesity is one of the most serious public health problems of the 21st century (World Health Organization [WHO], 2015). An excess of body fat has severe health consequences for children, making them more likely to develop diabetes or cardiovascular diseases or to have a premature death later in life (GBD 2015 Obesity Collaborators, 2017; Lavie, McAuley, Church, Milani, \& Blair, 2014; Ortega, Lavie, \& Blair, 2016). Likewise, physical fitness components (i.e. cardiorespiratory fitness, speed/agility, and musculoskeletal fitness) are considered important health-related markers already in youth, and may serve as potential protectors to reduce the harmful effects of fatness on health (Ortega, Ruiz, Castillo, \& Sjöström, 2008; Ruiz et al., 2009).

Since fatness and fitness are important predictors of health-related factors, it is of relevance to identify their common determinants. There is strong evidence supporting that genetics greatly determines both fatness and fitness, but less is known about the influence of environmental factors on these two parameters (Bray et al., 2009; Nightingale, Rudnicka, Owen, Cook, \& Whincup, 2011). 
Among several environmental factors, the socioeconomic status (SES) may play an important role by influencing lifestyle or facilitating access to health care; however, the available findings on the relationships between SES and fatness and fitness are inconclusive (Sandercock et al., 2017). A negative association has been consistently shown between the SES and childhood obesity (Bammann et al., 2013; Shrewsbury \& Wardle, 2008), whereas inconsistent results have been found between the SES and fitness in youth (Freitas et al., 2007; Sandercock et al., 2017).

The existing evidence of the relationship between the SES and fatness and fitness has focused mainly on school children and adolescents, however environmental factors have shown a greater influence already in preschoolers' lifestyle (Schmutz et al., 2017). Thus, it would be interesting to examine the mentioned relationship in the first years of life. To the best of our knowledge, scientific literature on preschoolers has mainly focused on examining the relationship between the SES and motor fitness (i.e. speed/agility, balance, coordination, etc.) (Barnett, Hinkley, Okely, \& Salmon, 2013; Bürgi et al., 2010; Morley, Till, Ogilvie, \& Turner, 2015; Ortega et al., 2015), without taking cardiorespiratory fitness and musculoskeletal fitness into account. Furthermore, to our knowledge, no studies have analysed the role of parental marital status in preschoolers' fatness and fitness. Thus, the present study has the aim 1) to analyse the association between the SES, measured by parental educational and occupational levels, with fatness (i.e. total adiposity and abdominal adiposity) and fitness (i.e. cardiorespiratory fitness, speed/agility, and musculoskeletal fitness) in preschoolers; and 2) to examine whether fatness and fitness levels differ according to the marital status.

\section{Methods}

\section{Participants}

The present cross-sectional study was performed under the framework of the PREFIT project (http://profith.ugr.es/pre fit) which is a multicentre project designed to assess anthropometry and physical fitness in a sample of preschool children geographically distributed across 10 towns/cities of Spain (Almería, Cádiz, Castellón, Cuenca, Granada, Las Palmas de Gran Canaria, Madrid, Palma de Mallorca, Zaragoza, and Vitoria-Gasteiz) (Cadenas-Sanchez et al., 2018, 2016; Ortega et al., 2015). An initial convenience sample of 3,179 healthy preschoolers aged 3-5 years old was recruited. The data collection was conducted from January 2014 to November 2015. For the present study, those participants who had complete and valid data on self-reported SES factors (i.e., educational and occupational levels), marital status, fatness, fitness, and the main covariates were included ( $n=2638,4.6 \pm 0.9$ years). Among them, 787 preschoolers were of 3 years-old, 885 of 4 yearsold and 966 of 5 years-old. All measurements were carried out by trained researchers of the PREFIT project.

One legal guardian or parent provided a written informed consent. The study protocol was performed in accordance with the ethical standards (Declaration of Helsinki revised in 2013) and was approved by the Review Committee for Research Involving Human Subjects at the University of Granada.

\section{Socioeconomic status}

\section{Parental educational level}

A questionnaire about the highest educational level achieved was filled in by both parents. A variable with three categories was computed for each parent: low (no education or primary school education), medium (secondary school education, upper-secondary school education, or technical training), and high (university education). Also, a parental combined variable was computed for the educational level: low (neither parent had university education), medium (one of the parents had university education), and high (both parents had university education) (Huppertz et al., 2017).

\section{Parental occupational level}

Both parents were asked to answer an open question concerning their current occupation. The answers of each parent were categorised following the International Standard Classification of Occupations (ISCO) and taking into account the Homemakers (11), and Unemployed (12) (International Labour Office, 2012). The ISCO categories were re-categorised as high (1 to 3), medium (4 to 8 ), and low (9 to 12). Also, a parental combined variable was computed for the occupational level: low (neither parent had a high occupational level), medium (one of the parents had a high occupational level), and high (both parents had a high occupational level).

\section{Marital status}

The marital status was self-reported by the parents using the following question: "What is your current marital status?" They chose 1 of 4 answers: single, married, divorced, or widowed. As in previous studies (Hesketh, Crawford, Salmon, Jackson, \& Campbell, 2007; Yannakoulia et al., 2008), the marital status was finally categorised as: single (1), married (2), divorced (3). Only 5 parents answered "widowed" and were excluded from the analyses.

\section{Fatness}

The weight $(\mathrm{kg})$ was measured using a SECA scale (869 scale, Hamburg, Germany; accuracy $0.05 \mathrm{~g}$ ) and the height $(\mathrm{cm})$ was measured using a stadiometer (SECA model 213, accuracy $0.10 \mathrm{~cm}$ ). The body mass index (BMI) was calculated as the weight $(\mathrm{kg})$ divided by the height squared $\left(\mathrm{m}^{2}\right)$. The waist circumference (WC) $(\mathrm{cm})$ was measured at the level of the umbilicus zone in the horizontal plane with a non-elastic tape (SECA model 200). The waist-to-height ratio $(\mathrm{WHtR})(\mathrm{cm} / \mathrm{m})$ was calculated as the $\mathrm{WC}$ expressed in centimetres divided by the height expressed in metres. All measurements were taken twice, and the mean was used in the analyses. 


\section{Physical fitness}

The physical fitness components were assessed using the feasible and reliable evidence-based physical FITness test battery in PREschool children: the PREFIT battery (http://profith. ugr.es/recursos-prefit?lang=en) (Cadenas-Sánchez et al., 2014; Cadenas-Sanchez et al., 2016; Ortega et al., 2015).

\section{Cardiorespiratory fitness}

The PREFIT 20-meters shuttle-run test (PREFIT 20m SRT) was used to assess cardiorespiratory fitness. This test has been specifically adapted from the original one (Léger, Mercier, Gadoury, \& Lambert, 1988) to be used in preschool children (Cadenas-Sánchez et al., 2014). The initial speed was $6.5 \mathrm{~km} /$ $\mathrm{h}^{-1}$, increasing $0.5 \mathrm{~km} / \mathrm{h}^{-1}$ every stage. The participants did this test once and always at the end of the fitness battery testing session. We registered the last completed lap, and we estimated $\mathrm{VO}_{2} \max (\mathrm{ml} / \mathrm{kg} / \mathrm{min})$ using the original equation of Leger (Léger et al., 1988) adapted to the PREFIT 20m SRT for preschoolers (Mora-Gonzalez et al., 2017).

\section{Speed/agility}

The $4 \times 10$-meters shuttle-run test $(4 \times 10 \mathrm{~m}$ SRT) was used to assess speed/agility. The participants ran back and forth four times between two parallel lines $10 \mathrm{~m}$ apart at the highest speed possible. The preschoolers did the test twice, and the best performance was registered for analyses (sec). In this test, a longer time indicates a poorer performance (i.e., slower and less agile).

\section{Musculoskeletal fitness}

The upper body muscular strength was assessed with the handgrip strength test $(H G)$ using a dynamometer (TKK 5001, Grip-A, Takei, Tokyo, Japan; range 0-100kg; accuracy $0.5 \mathrm{~kg}$ ). The optimal grip span was fixed at $4.0 \mathrm{~cm}$ (Sanchez-Delgado et al., 2015). The child performed the test twice, alternating with both hands, and the maximum score for each hand was recorded in kilograms $(\mathrm{kg})$ to compute the mean of both hands. To account for differences in body size, the absolute HG test was divided by body weight (relative HG strength).

The standing long-jump test (SLJ) was used to assess the lower body muscular power. In this test, the preschoolers had to jump as far as possible with their feet separated from each other. We registered the longest distance achieved, and we recorded the best of three attempts $(\mathrm{cm})$ as a relative measurement of lower muscular power.

The individual score of each test was standardized as follows: $z$-standardized value $=($ value - the sample mean $) / S D$. The $z$-score of musculoskeletal fitness was then calculated as the mean of the two standardized scores (relative HG strength + SLJ)/2.

\section{Statistical analysis}

A significant sex interaction was found only for the association between the paternal educational level and the $S \sqcup$ test $(P=0.025)$. Therefore, we studied the relationship between the paternal education and the S $\amalg$ test separately for boys and girls. Prior to all analyses, all outcomes were checked for normal distribution and all were normally distributed. To examine the differences between levels of each SES factor and marital status with respect to fatness and fitness outcomes, we conducted analyses of covariance (ANCOVA) adjusted by age and sex, with pairwise comparisons with Bonferroni adjustments.

Cohen's $d$ (standardized mean difference) was assessed as a measure of the magnitude of effect. Taking into account the cut-off established by Cohen, the effect size (Cohen's $d$ ) can be small $(d=0.2)$, medium ( $d=0.5)$ or large $(d=0.8)$ (Nakagawa \& Cuthill, 2007). In addition to the main analyses previously described, additional ANCOVA analyses were performed to examine whether the results obtained by the main analyses remained constant after adjusting SES and fatness models by marital status and standing long jump test, and SES and fitness models by marital status and BMI.

The binary logistic regression was used to obtain the odds ratio (OR) and $95 \%$ confidence intervals $(95 \% \mathrm{Cl})$ of being obese for every level of paternal education and occupation adjusting by sex and age. BMI was categorised in non-overweight-obese ( $\mathrm{n}=2087$ ) vs. overweight-obese ( $n=560$ ), according to the Cole \& Lobstein' cut-offs (Cole \& Lobstein, 2012). Only a $4.6 \%$ of the total sample fell into "underweight category" so they were included together with normal-weight preschoolers into the non-overweightobese category. Since musculoskeletal fitness was the component with the strongest relation to the SES, the same analysis was performed to obtain the OR of having low musculoskeletal fitness. In this case, a z-score of musculoskeletal fitness was categorised as low musculoskeletal fitness (i.e. participants below the age-specific and sexspecific 20th percentile of the sample, $n=528$ ) vs. middlehigh musculoskeletal fitness (i.e. all rest of participants above the 20th percentile, $n=2119$ ). The use of the 20th percentile has been previously used (Ortega et al., 2016; Ortega, Ruiz, Labayen, Lavie, \& Blair, 2017). For all of the analyses, we set a significance level of $P<0.05$. All the statistical procedures were performed using the SPSS software for Windows (version 22.0, IBM Corporation).

\section{Results}

\section{Differences in fatness across parental SES levels}

The descriptive characteristics of the preschool children ( $n=2638$ ) are shown in Table 1. We found significant differences in BMI, WC, and WHtR across both paternal and maternal educational levels $(P<0.05)$ (Table 2$)$. Significant differences were also found in BMl across both paternal and maternal occupational levels $(P<0.05)$, and in WHtR across paternal occupational levels $(P=0.004)$. After additional analyses, all these differences were considered statistically independent of marital status and fitness, except the differences in WHtR across paternal educational levels as the significance disappeared $(P \geq 0.05)$. We observed small effect sizes (Cohen's $d \leq 0.2)$ for the previous analyses with significant differences.

Those participants with both parents with high or medium educational levels had lower odds of being obese compared with those with both parents with low educational levels $(\mathrm{OR}=0.67,95 \% \mathrm{Cl}$ : 0.541-0.853; $\mathrm{OR}=0.72$, 95\% Cl: 0.576-0.914, respectively) (Figure 1). Similarly, 
Table 1. Descriptive characteristics of the sample.

\begin{tabular}{|c|c|c|c|}
\hline & All $(n=2638)$ & Boys $(n=1393)$ & Girls $(n=1245)$ \\
\hline Age (years) & $4.6 \pm 0.9$ & $4.59 \pm 0.87$ & $4.59 \pm 0.88$ \\
\hline \multicolumn{4}{|l|}{ Socioeconomic status } \\
\hline \multicolumn{4}{|l|}{ Paternal education [n (\%)] } \\
\hline Low & 255 (9.7\%) & 137 (9.8\%) & $118(9.5 \%)$ \\
\hline Medium & 1383 (52.4\%) & $716(51.4 \%)$ & 667 (53.6\%) \\
\hline High & $1000(37.9 \%)$ & 540 (38.8\%) & 460 (36.9\%) \\
\hline \multicolumn{4}{|l|}{ Maternal education $[\mathrm{n}(\%)]$} \\
\hline Low & $451(17.1 \%)$ & $251(18.0 \%)$ & $200(16.1 \%)$ \\
\hline Medium & $910(34.5 \%)$ & $476(34.2 \%)$ & $434(34.9 \%)$ \\
\hline High & $1277(48.4 \%)$ & $666(47.8 \%)$ & $611(49.1 \%)$ \\
\hline \multicolumn{4}{|l|}{ Paternal occupation [n (\%)] } \\
\hline Low & $538(20.4 \%)$ & 277 (19.9\%) & $261(21.0 \%)$ \\
\hline Medium & $1110(42.1 \%)$ & $582(41.8 \%)$ & $528(42.4 \%)$ \\
\hline High & $990(37.5 \%)$ & $534(38.3 \%)$ & $456(36.6 \%)$ \\
\hline \multicolumn{4}{|l|}{ Maternal occupation [n (\%)] } \\
\hline Low & $833(31.6 \%)$ & 446 (32.0\%) & 387 (31.1\%) \\
\hline Medium & $833(31.6 \%)$ & $454(32.6 \%)$ & $379(30.4 \%)$ \\
\hline High & $972(36.8 \%)$ & 493 (35.4\%) & 479 (38.5\%) \\
\hline \multicolumn{4}{|l|}{ Marital status [n (\%)] ${ }^{*}$} \\
\hline Single & $433(16.4 \%)$ & $222(15.9 \%)$ & $211(16.9 \%)$ \\
\hline Married & $2104(79.8 \%)$ & 1127 (80.9\%) & 977 (78.5\%) \\
\hline Divorced & 101 (3.8\%) & $44(3.2 \%)$ & $57(4.6 \%)$ \\
\hline \multicolumn{4}{|l|}{ Fatness } \\
\hline Weight $(\mathrm{kg})$ & $19.0 \pm 3.7$ & $19.2 \pm 3.9$ & $18.7 \pm 3.5$ \\
\hline Height $(\mathrm{cm})$ & $106.9 \pm 7.5$ & $107.5 \pm 7.5$ & $106.3 \pm 7.4$ \\
\hline $\mathrm{BMI}\left(\mathrm{kg} / \mathrm{m}^{2}\right)$ & $16.5 \pm 1.7$ & $16.5 \pm 1.8$ & $16.5 \pm 1.7$ \\
\hline Waist circumference $(\mathrm{cm})$ & $53.2 \pm 5.0$ & $53.0 \pm 5.0$ & $53.4 \pm 5.0$ \\
\hline Waist-to-height ratio & $0.50 \pm 0.04$ & $0.49 \pm 0.04$ & $0.50 \pm 0.04$ \\
\hline \multicolumn{4}{|l|}{ Fitness } \\
\hline Handgrip strength test $(\mathrm{kg})$ & $7.0 \pm 2.5$ & $7.4 \pm 2.5$ & $6.6 \pm 2.3$ \\
\hline HG/body weight & $0.37 \pm 0.10$ & $0.38 \pm 0.10$ & $0.35 \pm 0.10$ \\
\hline Standing long jump test $(\mathrm{cm})$ & $74.0 \pm 22.2$ & $77.4 \pm 21.9$ & $70.2 \pm 22.0$ \\
\hline $4 \times 10 m$ SRT (sec) & $16.8 \pm 2.5$ & $16.5 \pm 2.4$ & $17.2 \pm 2.6$ \\
\hline PREFIT 20m SRT (shuttles) & $19.9 \pm 11.6$ & $21.4 \pm 12.2$ & $18.2 \pm 10.6$ \\
\hline PREFIT $20 \mathrm{~m}$ SRT (estimated $\mathrm{VO}_{2} \mathrm{max}, \mathrm{mL} / \mathrm{kg} / \mathrm{min}$ ) $\dagger$ & $49.1 \pm 1.7$ & $49.3 \pm 1.7$ & $48.8 \pm 1.7$ \\
\hline
\end{tabular}

The values are means \pm standard deviation unless otherwise indicated. *The sample size for marital status descriptive data was $\mathrm{n}=2845$ preschoolers (4.6 \pm 0.9 years; $52.5 \%$ boys). $+T_{\text {The }} \mathrm{VO}_{2} \max (\mathrm{ml} / \mathrm{kg} / \mathrm{min}$ ) was estimated from the completed stages and the age using the original equation of Leger (Léger et al., 1988) adapted to the PREFIT 20m SRT for preschoolers by Mora et al. (Mora-Gonzalez et al., 2017). BMI = Body mass index; HG = Handgrip strength test; SRT $=$ Shuttle-run test.

Table 2. Differences in fatness across parental educational and occupational levels in preschoolers $(n=2638)$.

\begin{tabular}{|c|c|c|c|}
\hline & BMI $\left(\mathrm{kg} / \mathrm{m}^{2}\right)$ & Waist circumference $(\mathrm{cm})$ & Waist-to-height ratio * \\
\hline \multicolumn{4}{|l|}{ Educational status } \\
\hline \multicolumn{4}{|l|}{ Paternal education } \\
\hline Low (Low-Medium) & $16.6 \pm 0.11(0.04)$ & $53.3 \pm 0.29(0.00)$ & $50.2 \pm 0.23^{\mathrm{a}}(0.07)$ \\
\hline Medium (Medium-High) & $16.6 \pm 0.05^{\mathrm{a}}(0.11)$ & $53.3 \pm 0.13^{\mathrm{a}}(0.10)$ & $49.9 \pm 0.10^{b}(0.13)$ \\
\hline High (Low-High) & $16.3 \pm 0.06^{\mathrm{a}}(0.15)$ & $52.9 \pm 0.15^{\mathrm{a}}(0.10)$ & $49.6 \pm 0.12^{\mathrm{a}, \mathrm{b}}(0.20)$ \\
\hline Overall $P$-value & 0.005 & 0.039 & 0.001 \\
\hline \multicolumn{4}{|l|}{ Maternal education } \\
\hline Low (L-M) & $16.7 \pm 0.08^{\mathrm{a}}(0.09)$ & $53.7 \pm 0.22^{\mathrm{a}}(0.09)$ & $50.3 \pm 0.18^{\mathrm{a}}(0.10)$ \\
\hline Medium (M-H) & $16.5 \pm 0.06^{\mathrm{b}}(0.10)$ & $53.3 \pm 0.15(0.09)$ & $49.9 \pm 0.12(0.11)$ \\
\hline High (L-H) & $16.4 \pm 0.05^{\mathrm{a}, \mathrm{b}}(0.20)$ & $52.9 \pm 0.13^{\mathrm{a}}(0.18)$ & $49.5 \pm 0.10^{\mathrm{a}}(0.21)$ \\
\hline Overall P-value & 0.001 & 0.004 & 0.001 \\
\hline \multicolumn{4}{|l|}{ Occupational status } \\
\hline \multicolumn{4}{|l|}{ Paternal occupation } \\
\hline Low (L-M) & $16.6 \pm 0.08^{\mathrm{a}}(0.04)$ & $53.4 \pm 0.20(0.03)$ & $50.0 \pm 0.16^{\mathrm{a}}(0.02)$ \\
\hline Medium (M-H) & $16.5 \pm 0.05(0.10)$ & $53.3 \pm 0.14(0.08)$ & $49.9 \pm 0.11^{\mathrm{b}}(0.12)$ \\
\hline High $(\mathrm{L}-\mathrm{H})$ & $16.4 \pm 0.06^{\mathrm{a}}(0.13)$ & $52.9 \pm 0.15(0.11)$ & $49.5 \pm 0.12^{\mathrm{a}, \mathrm{b}}(0.15)$ \\
\hline Overall $P$-value & 0.015 & 0.060 & 0.004 \\
\hline \multicolumn{4}{|l|}{ Maternal occupation } \\
\hline Low (L-M) & $16.5 \pm 0.06(0.03)$ & $53.4 \pm 0.16(0.02)$ & $49.9 \pm 0.13(0.01)$ \\
\hline Medium $(\mathrm{M}-\mathrm{H})$ & $16.6 \pm 0.06^{\mathrm{a}}(0.12)$ & $53.3 \pm 0.16(0.07)$ & $49.9 \pm 0.13(0.07)$ \\
\hline High (L-H) & $16.4 \pm 0.06^{\mathrm{a}}(0.09)$ & $52.9 \pm 0.15(0.09)$ & $49.6 \pm 0.12(0.08)$ \\
\hline Overall $P$-value & 0.027 & 0.110 & 0.146 \\
\hline
\end{tabular}

The values are adjusted means \pm standard error with a Bonferroni adjustment. Effects size statistics for pair comparisons are expressed as Cohen's $d$ in parenthesis. Values sharing a common superscript show significant post-hoc differences between pairs at $P<0.05$. The analysis of covariance (ANCOVA) was adjusted by sex and age. Significant differences are highlighted in bold. *The waist-to-height ratio $(\mathrm{cm} / \mathrm{m})$ was calculated as the waist circumference expressed in centimetres divided by the height expressed in metres. $\mathrm{BMI}=$ Body mass index. 

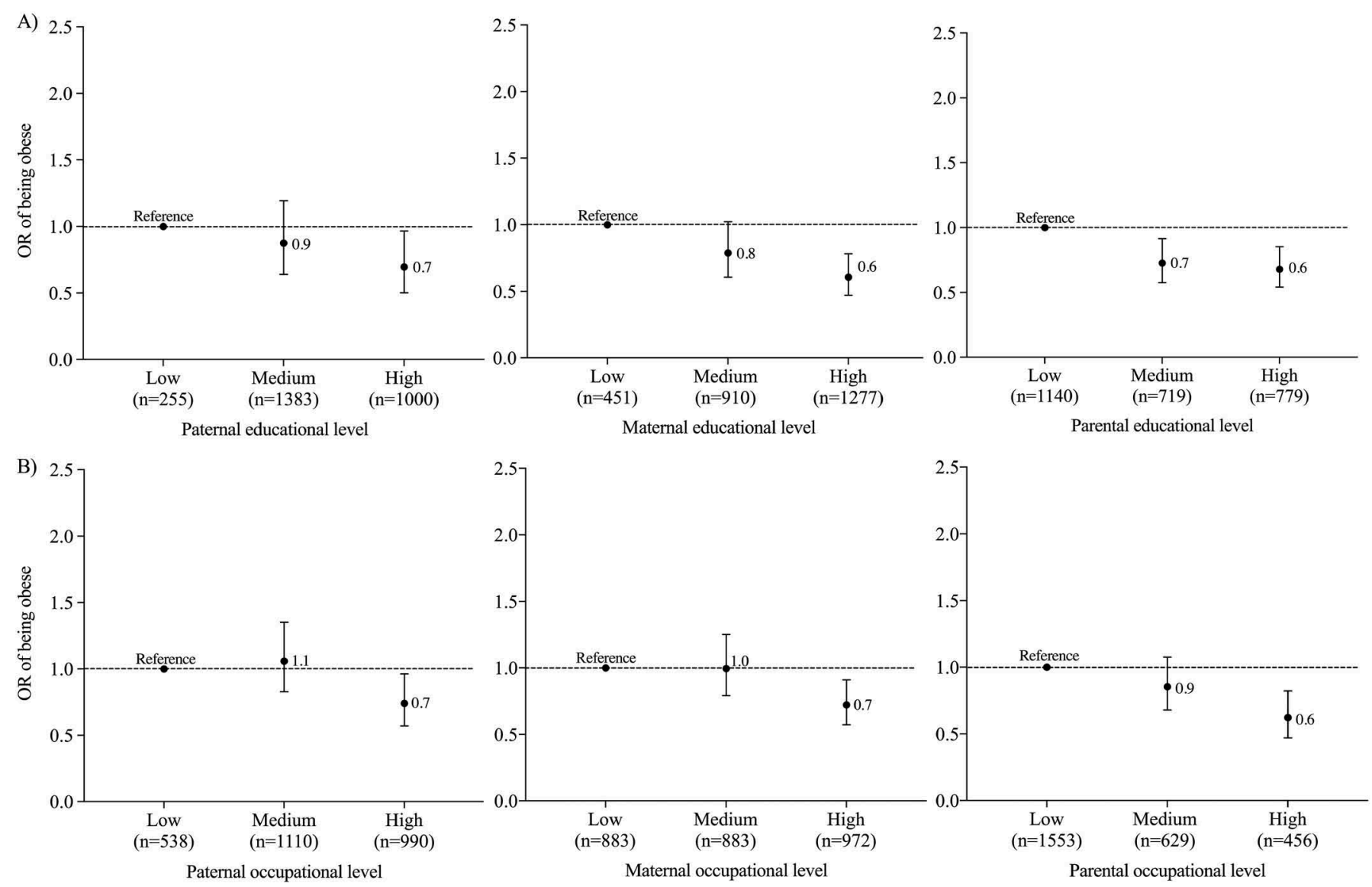

Figure 1. Odds ratio $(95 \% \mathrm{Cl})$ for the relation of parental educational and occupational levels to being overweight-obese $(\mathrm{n}=560$; $\mathrm{non}$-overweight-obese $\mathrm{n}=2078$ ) The logistic regression models were adjusted by sex and age. Low educational and occupational levels are set as the reference level (i.e., value 1), so that the odds ratio is presented for the medium and high level compared with the low level.

children whose parents had high occupational levels had lower odds of being obese compared to those with low parental occupational levels $(\mathrm{OR}=0.62,95 \% \mathrm{Cl}$ : $0.470-0.823)$. The results were similar when the data were examined separately for maternal and paternal SES levels (Figure 1).

\section{Differences in physical fitness across parental SES levels}

We found significant differences in relative HG strength and the $\mathrm{SLJ}$ test across both paternal and maternal educational levels $(P<0.05)$ (Table 3$)$. No significant differences among educational categories were found in the rest of the physical fitness tests $(P \geq 0.05)$, except for the estimated $\mathrm{VO}_{2} \max$ from the PREFIT $20 \mathrm{~m} \mathrm{SRT}(P=0.046)$. We found significant differences in relative $H G$ strength and in the SLJ test across maternal occupational levels, and also in the SLJ test and the $4 \times 10 \mathrm{~m}$ SRT across paternal levels $(P<0.05)$. After additional analyses, all these differences were considered statistically independent of marital status and fatness, except the differences in relative HG strength across maternal educational levels as the significance disappeared $(P>0.05)$. On the other hand, after this adjustment all the analyses between SES and absolute HG turned into significant $(P<0.05)$. We observed small effect sizes (Cohen's $d \leq 0.2$ ) for the previous analyses with significant differences.

Preschoolers whose parents had high educational levels had lower odds of having low musculoskeletal fitness compared to their peers with low parental educational levels $(O R=0.69,95 \%$ Cl: 0.545-0.874) (Figure 2). Similar results were found for high paternal educational levels. Preschoolers with parents with high occupational levels had lower odds of having a low musculoskeletal fitness compared to those with low occupational levels $(\mathrm{OR}=0.75,95 \% \mathrm{Cl}: 0.571-0.989)$. A similar pattern was observed for high and medium paternal and maternal occupational levels with respect to their peers with low parental occupational levels (Figure 2).

\section{Differences in fatness and physical fitness according to marital status}

According to the marital status, those preschoolers whose parents were married completed a higher number of laps in the PREFIT $20 \mathrm{~m}$ SRT than their peers whose parents were single $(P=0.005)$ (Figure 3). Similar results were found for the estimated $\mathrm{VO}_{2} \mathrm{max}$ from the PREFIT $20 \mathrm{~m}$ SRT (data not shown). We found no significant differences for the rest of 
Table 3. Differences in fitness across parental educational and occupational levels in preschoolers $(n=2638)$.

\begin{tabular}{|c|c|c|c|c|c|c|}
\hline & $\begin{array}{l}\text { Handgrip } \\
\text { strength } \\
\text { test }(\mathrm{kg})\end{array}$ & HG/body weight & $\begin{array}{l}\text { Standing long jump } \\
\text { test }(\mathrm{cm})^{*}\end{array}$ & $\begin{array}{c}4 \times 10 \mathrm{~m} \text { SRT } \\
(\mathrm{sec}) \dagger\end{array}$ & $\begin{array}{l}\text { PREFIT 20m SRT } \\
\text { (shuttles) }\end{array}$ & $\begin{array}{c}\text { PREFIT 20m SRT } \\
\text { (estimated } \mathrm{VO}_{2} \text { max) }\end{array}$ \\
\hline \multicolumn{7}{|l|}{ Educational status } \\
\hline Medium (Medium-High) & $7.0 \pm 0.05(0.08)$ & $0.36 \pm 0.002^{\mathrm{a}}(0.12)$ & $73.4 \pm 0.45^{\mathrm{b}}(0.12)$ & $16.9 \pm 0.05(0.05)$ & $19.5 \pm 0.26(0.05)$ & $49.0 \pm 0.04(0.04)$ \\
\hline High (Low-High) & $7.1 \pm 0.06(0.11)$ & $0.37 \pm 0.003^{\mathrm{a}}(0.11)$ & $75.4 \pm 0.53^{\mathrm{a}, \mathrm{b}}(0.20)$ & $16.8 \pm 0.06(0.13)$ & $20.1 \pm 0.31(0.09)$ & $49.1 \pm 0.05(0.09)$ \\
\hline Overall $P$-value & 0.070 & 0.010 & 0.002 & 0.119 & 0.076 & 0.114 \\
\hline Medium $(\mathrm{M}-\mathrm{H})$ & $7.0 \pm 0.06(0.05)$ & $0.36 \pm 0.003(0.09)$ & $73.3 \pm 0.55(0.09)$ & $16.9 \pm 0.06(0.04)$ & $19.3 \pm 0.32(0.08)$ & $49.0 \pm 0.05(0.09)$ \\
\hline High (L-H) & $7.1 \pm 0.05(0.07)$ & $0.37 \pm 0.002^{\mathrm{a}}(0.16)$ & $74.9 \pm 0.47(0.12)$ & $16.8 \pm 0.05(0.03)$ & $20.1 \pm 0.27(0.03)$ & $49.1 \pm 0.04(0.04)$ \\
\hline Overall $P$-value & 0.295 & 0.016 & 0.029 & 0.599 & 0.084 & 0.046 \\
\hline \multicolumn{7}{|l|}{ Occupational status } \\
\hline \multicolumn{7}{|l|}{ Paternal occupation } \\
\hline Low (L-M) & $7.0 \pm 0.07(0.03)$ & $0.36 \pm 0.004(0.04)$ & $71.6 \pm 0.72^{\mathrm{a}, \mathrm{b}}(0.14)$ & $17.1 \pm 0.08^{\mathrm{a}, \mathrm{b}}(0.15)$ & $19.4 \pm 0.42(0.07)$ & $49.0 \pm 0.07(0.05)$ \\
\hline Medium $(\mathrm{M}-\mathrm{H})$ & $7.0 \pm 0.05(0.07)$ & $0.37 \pm 0.003(0.06)$ & $73.9 \pm 0.50^{\mathrm{a}}(0.09)$ & $16.8 \pm 0.05^{\mathrm{a}}(0.02)$ & $20.1 \pm 0.29(0.02)$ & $49.1 \pm 0.05(0.02)$ \\
\hline High (L-H) & $7.1 \pm 0.06(0.09)$ & $0.37 \pm 0.003^{\mathrm{a}}(0.13)$ & $74.7 \pm 0.53^{\mathrm{b}}(0.15)$ & $16.8 \pm 0.06(0.08)$ & $20.0 \pm 0.31(0.01)$ & $49.1 \pm 0.05(0.02)$ \\
\hline Overall $P$-value & 0.131 & 0.013 & 0.001 & 0.163 & 0.681 & 0.705 \\
\hline
\end{tabular}

The values are adjusted means \pm standard error with a Bonferroni adjustment. Effects size statistics for pair comparisons are expressed as Cohen's $d$ in parenthesis. Values sharing a common superscript show significant post-hoc differences between pairs at $P<0.05$. The analysis of covariance (ANCOVA) was adjusted by sex and age. Significant differences are highlighted in bold. * There was a significant interaction between sex and paternal education with the standing long jump test $(P=0.025)$. There was a significant difference between low $(66.1 \pm 1.50)$, medium $(69.4 \pm 0.63)$, and high $(72.4 \pm 0.76)$ paternal education for the standing long jump test in girls $(P<0.001)$, whereas no significant differences were found between low $(77.2 \pm 1.45)$, medium $(76.9 \pm 0.63)$, and high (78.1 \pm 0.73$)$ paternal education in boys $(P=0.477)$. $†$ Higher values of speed-agility mean lower level of fitness. + +Calculated using Mora et al. equation for estimating $\mathrm{VO}_{2}$ max from stages and age in preschoolers (Mora-Gonzalez et al., 2017).

$\mathrm{HG}=$ handgrip strength test; SRT = shuttle-run test.
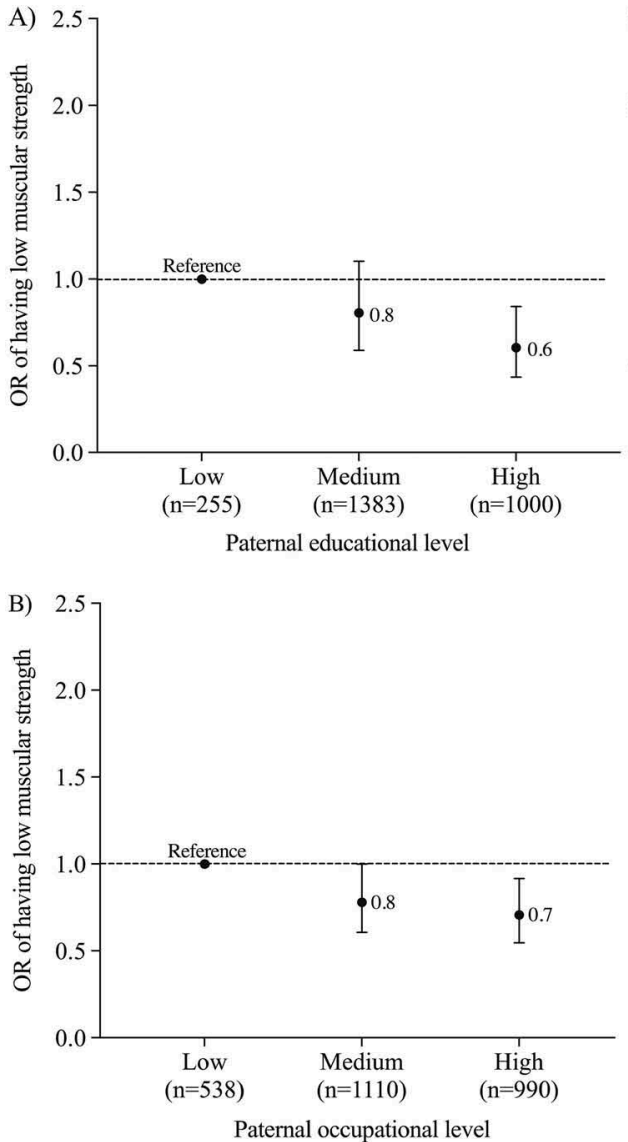

Paternal occupational level

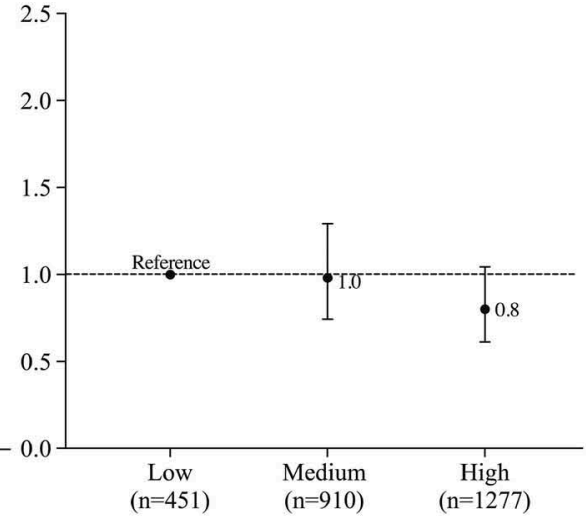

Maternal educational level

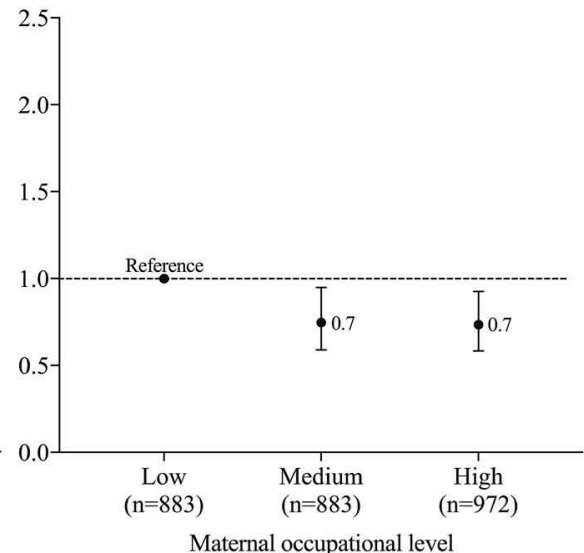

Maternal occupational level

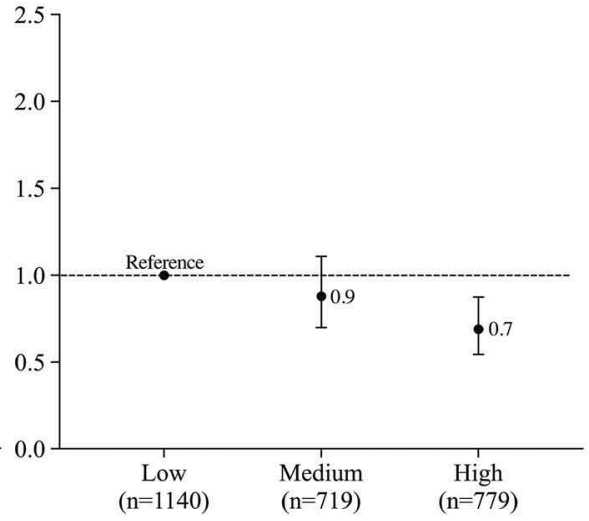

Parental educational level

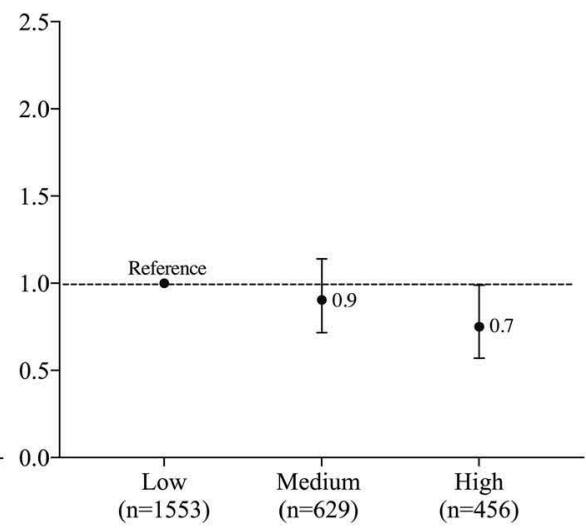

Parental occupational level

Figure 2. Odds ratio $(95 \% \mathrm{Cl})$ for the relation of parental educational and occupational levels with having a low muscular strength $(\mathrm{n}=526$; Middle-high muscular strength, $n=2112$ ). The logistic regression models were adjusted by sex and age. Low educational and occupational levels are set as the reference level (i.e., value 1), so that the odds ratio is presented for the medium and high level compared with the low level. 

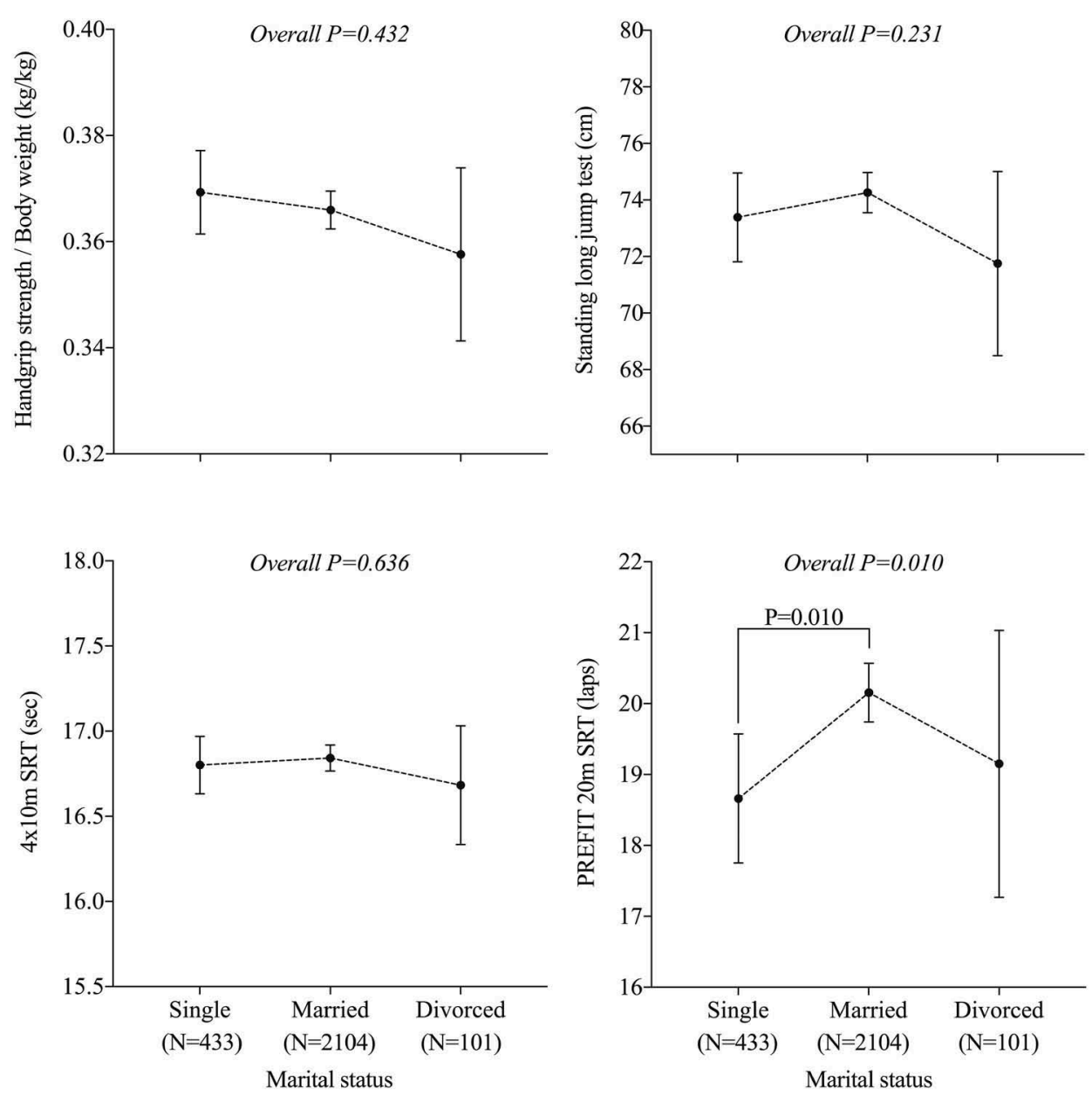

Figure 3. Differences in physical fitness components with respect to the marital status. The ANCOVA analyses were adjusted by sex and age. The dots indicate the estimated mean, and the error bars represent $95 \%$ confidence intervals. SRT = shuttle-run test.

physical fitness tests with respect to the marital status (all $P \geq 0.05$ ). Likewise, no significant differences were observed in fatness indicators (data not shown). We observed small effect sizes (Cohen's $d \leq 0.2$ ) for the previous analyses with significant differences.

\section{Discussion}

\section{Main findings}

The main findings of the present study were that: 1) Preschool children whose parents had a higher SES had lower fatness independently of marital status and fitness; 2) Similarly, preschool children with parents with high educational and occupational levels performed better in the musculoskeletal fitness tests expressed in relative terms (i.e., relative HG strength and SLJ) independently of marital status and fatness. In this case the differences were slightly larger among the occupational levels than among the educational ones (except for paternal occupational levels and relative HG strength); 3) We did not observe differences either in fatness or fitness indicators among occupational and educational levels, except for cardiorespiratory fitness by estimated $\mathrm{VO}_{2} \max$ (PREFIT $20 \mathrm{~m} \mathrm{SRT}$ ) among maternal educational levels, and for speed/agility
(4 x 10 m SRT) across paternal occupational levels; 4) We observed no consistent differences between mothers and fathers according to their education and occupation and in relation to preschoolers' fatness and fitness; 5) Preschoolers whose parents (individually and both together) had high SES levels may have lower odds of becoming obese and of having a low musculoskeletal fitness compared to those of low SES; 6) Preschoolers whose parents were married had higher cardiorespiratory fitness (i.e. shuttles in PREFIT $20 \mathrm{~m}$ SRT) than those whose parents were single; 7) In general, we observed a small effect size (Cohen's $d \leq 0.2$ ) for the significant differences in fatness and physical fitness across SES outcomes.

\section{Fatness across parental SES levels}

In contrast to the significant differences found in the present study in fatness markers across levels of SES in Spanish preschoolers, a study with Swiss preschoolers found no significant differences in BMI and skinfolds (Bürgi et al., 2010). However, other studies carried out in older children do support our findings (Kobzová, Vignerová, Bláha, Krejcovský, \& Riedlová, 2004; Ortega et al., 2013). For instance, one study showed that BMI was lower in those children whose parents had high educational levels (Kobzová et al., 2004). The methodological differences and 
disparities between socio-cultural environments of different places within Europe (Lobstein \& Frelut, 2003) might explain the contradictory results. Furthermore, the influence of the SES on preschoolers' fatness might be due to the role that the maternal educational level plays when selecting foods for their children, since the most educated mothers may have greater knowledge and, therefore, select healthier food (Cutting, Fisher, Grimm-Thomas, \& Birch, 1999). However, the traditional view of the mother as the person who plays the major role in raising the children might be changing toward a more equal distribution of tasks regarding home and child care (Wang \& Sweetman, 2013). This notion seems to be supported by our findings which showed significant associations between both paternal and maternal SES factors and fatness and fitness.

There is a growing body of evidence suggesting that the SES is a risk factor of childhood obesity (Shrewsbury \& Wardle, 2008; Wu et al., 2015). In the present study, preschoolers whose father, mother, or both together presented a high SES had around $30-40 \%$ lower risk of becoming obese compared to their peers of low SES. These findings are consistent with a recent metaanalysis that found that low SES was associated with a $10 \%$ higher risk of overweight and a $41 \%$ higher risk of obesity in children aged 0-15 years old (Wu et al., 2015). Interestingly, another finding from this meta-analysis was that parental educational levels were more consistently associated with childhood overweight and obesity than other SES factors (e.g., family income, living space, etc.). This is also supported by another study carried out in preschoolers where overweight/obesity has been shown to be more prevalent among preschoolers of lower SES (Kitsantas \& Gaffney, 2010). This may be due to the fact that parental educational levels seem to influence the whole family's lifestyle and activity-related beliefs, and this is directly related to more or less healthy lifestyles (Drewnowski \& Specter, 2004; Winkleby, Kraemer, Ahn, \& Varady, 1998). Further, parental perceptions on the weight status of their preschool kids could vary depending on the SES status of the family as has been shown by a study where low maternal education was associated with a failure to perceive their preschoolers as overweight (Baughcum, Chamberlin, Deeks, Powers, \& Whitaker, 2000).

\section{Physical fitness across parental SES levels}

To the best of our knowledge, this is the first study to investigate the association between the SES with main health-related physical fitness components (Caspersen, Powell, \& Christenson, 1985) in preschoolers (i.e. 3-5-year-olds), since other studies that examined this association had only focused on motor skills and in 4-5-year-old preschoolers (Barnett et al., 2013; Bürgi et al., 2010). In contrast to our results in the $4 \times 10 \mathrm{~m} \mathrm{SRT} \mathrm{(i.e.,} \mathrm{speed/}$ agility) across educational levels, in the previously-mentioned study with Swiss preschoolers those with higher educated parents were more agile than those with lower educated parents (Bürgi et al., 2010). The different tests, the different methods to assess the educational level, and the multi-ethnic and cultural differences might explain the different results.

Although in the present study musculoskeletal fitness seems to be the strongest fitness component related to the SES in preschoolers, studies in older children have shown contradictory findings (Freitas et al., 2007; Imhof et al., 2016; Lämmle, Worth, \& Bös, 2012). In contrast to our results, an investigation with Portuguese youth reported higher lower body muscular power for the lowest SES level group in boys (Freitas et al., 2007). This study also found a negative relationship between the SES and cardiorespiratory fitness, but a positive association between the SES and speed/agility what concurs with our findings. Another study with 358 Swiss first graders found a positive association between parental educational level and lower body muscular power but no association with speed/agility (Imhof et al., 2016). Although we found no differences in cardiorespiratory fitness as measured by shuttles, we did find differences in this component as measured by estimated $\mathrm{VO}_{2}$ max across the maternal educational levels. This concurs with the previously-mentioned study also showing that with a higher combined education of both parents, a better performance was achieved in the $20 \mathrm{~m}$ SRT (Imhof et al., 2016). Whereas we observed an overall small effect size $(d \leq 0.2)$ for the differences in fitness across educational levels, this study found medium and large effect sizes for the differences in lower body muscular power test ( $d=0.5$ ) and the $20 \mathrm{~m} \mathrm{SRT}(d=0.8)$, respectively. Despite the inconsistent findings, several positive associations have been found between the SES and fitness, which may be explained by the fact that a higher SES could allow families to have an easier access to extracurricular sport activities, as well as to have a greater awareness of the importance of having a healthier lifestyle (Jiménez Pavón et al., 2010).

Another important finding of our study was that absolute HG did not show significant differences across SES levels. However, when $\mathrm{HG}$ was expressed in relation to body weight (i.e., relative HG strength) or when an additional adjustment by BMI was made, high SES levels were associated with higher absolute HG levels. These findings are in agreement with previous evidence suggesting that the SES-related differences in body size might explain some of the associations between the SES levels and fitness (Jiménez Pavón et al., 2010; Sandercock et al., 2017). Thus, it has been shown that the relative-to-body weight measurement of HG predicts different health outcomes more accurately than the absolute HG (Artero et al., 2011; Steene-Johannessen, Anderssen, Kolle, \& Andersen, 2009). This highlight the importance of taking into account the anthropometric characteristics of the sample in further studies (Jiménez Pavón et al., 2010; Otero et al., 2016; Sandercock et al., 2017).

In our study, having a high SES is related to having a lower risk of having low musculoskeletal fitness. Low musculoskeletal fitness during childhood and adolescence may increase the odds of having major causes of death in the young adulthood (Ortega, Silventoinen, Tynelius, \& Rasmussen, 2012). Individuals who have musculoskeletal fitness seem to be those who have families with lower SES and, in order to improve this relationship, they should be encouraged to engage in exercise and other forms of physical activity.

Fatness and physical fitness according to marital status

In our study, no significant differences were found in fatness across categories of marital status. However, several studies in children 
showed that those living in single-parent homes had a higher BMI than their peers living with both parents together (Hesketh et al., 2007; Yannakoulia et al., 2008). This association may grew stronger with age (Hesketh et al., 2007). These findings might explain our non-significant differences between marital status and fatness, since the impact of the marital status on the weight status could require longer exposure over time. On the other hand, and from all the fitness components, we found that preschoolers whose parents were married had a higher cardiorespiratory fitness than their peers whose parents were single. This could be due to the notion that, in general, families who function more cohesively (i.e., married parents) may generate a positive environment that facilitates the engagement of children in physical activity activities (Trost \& Loprinzi, 2011). Also, due to practical reasons, mono-parental families might have more time limitations to take children to afterschool sport activities or playgrounds than when there are two parents involved, since house and children care duties can be distributed between both parents instead. Further studies analysing the influence of the marital status on fatness and fitness levels are needed to clarify how family structure might influence important health outcomes, such as fatness and fitness.

\section{Limitations and strengths}

A small effect size was observed for the differences in fitness and fatness across SES levels that could be due to the multifactorial aetiology of preschoolers fitness and fatness status. The fact that we did not find a medium or even large effect by a single SES factor could be due to the variety of other heritable and environmental factors influencing. Thus, although medium or large effect sizes were observed in other studies also comparing fatness and fitness between different SES levels, (Imhof et al., 2016; Jiménez Pavón et al., 2010), in one of these studies the medium effect size was reduced to a small effect after controlling for other environmental factors such as household income and migrant background. In the present study, there is a lack of information about essential factors such as family income or living space or about important potential confounders as an objective measure of physical activity patterns of the sample. Another limitation was the causality of the association of fatness and fitness with SES which cannot be determined due to the cross-sectional design of the study. The use of anthropometrics as proxy of fatness instead of using more accurate body composition methods can be also considered as a limitation. On the other hand, the main strength of this study was the fact that, to the best of our knowledge, this was the first study to investigate the relationship between the SES and main health-related physical fitness components in a sample of 3-5-year-old preschool children. Another strength was the relatively large and geographically distributed (i.e., north, centre, and south, and the two island regions) sample of Spanish preschoolers. Furthermore, the sampling of the PREFIT Project includes 10 different cities all over Spain, which allows covering physical fitness and body composition data from preschoolers belonging to many parts of the country. The established validity and reliability of the PREFIT test battery used in the present research is a strength of the study (Cadenas-Sánchez et al., 2014; Cadenas-Sanchez et al., 2016; Mora-Gonzalez et al., 2017; Ortega et al., 2015).

\section{Practical implications}

We believe that the findings obtained in the present study have several practical implications that must be addressed: 1) Fatness and fitness are explained by an important genetic contribution and also by multiple environmental factors (e.g., perinatal factors, nutrition, exercise...). However, our results suggest that SES factors (i.e., parental educational and occupational levels) may have also a contribution to preschoolers' fatness and fitness. However, the fact that we did not find a medium or large effect by a single SES factor may be due to the wide variety of other heritable and environmental factors that could be influencing; 2 ) In addition, there may be a chance that the potential influence of SES on fatness and fitness could be amplified over time, i.e., if SES has an influence on individuals' fatness and fitness already after 3 years of life, such influence could potentially enhances the differences between children from low and high SES families as they grow older. Longitudinal studies will confirm or contrast this hypothesis; 3) Finally, we believe that in some cases the significant differences observed between SES groups could have practical implications. As an example, the difference in standing long jump in a preschool child from a father with low occupation al level is $71.6 \mathrm{~cm}$ vs. $76.0 \mathrm{~cm}$ in a child from a father with high occupational level, which corresponds to a difference of $4.4 \mathrm{~cm}$ in average. This represents nearly a $6 \%$ difference proportionally to the average jump of the population studied, what could be considered as a meaningful difference; 4) The findings of the present study indicate that already at early ages those who have a low SES have higher odds of being obese. This should be taken into account by public policies who should carry out educational programmes to help low SES families palliate these odds.

\section{Conclusion}

The results suggest that a high SES level seems to positively influence fatness and fitness outcomes, yet musculoskeletal fitness might be the health-related physical fitness component most strongly influenced by the SES. Furthermore, a low SES, as measured by either educational or occupational factors, seems to be a risk factor of becoming obese or having low musculoskeletal fitness in preschoolers. Both paternal and maternal SES levels seem to relate similarly to fatness and fitness levels of their children, which might be influenced by a growing equality regarding their presence in the household. In this sense, the marital status was solely associated to cardiorespiratory fitness. Public policies should carry out educational programmes in order to educate parents on how to keep a healthy lifestyle, so that they can transmit it to their children. Educating parents seems important already at preschool ages. Furthermore, the SES needs to be considered as a key factor in interventions targeted to reduce inequalities in health, and they should focus on the community level such as the school setting.

\section{Acknowledgments}

We would like to thank the preschool and children students, parents, and teachers for their participation in this study. We are grateful to Ms. Carmen Sainz-Quinn for assistance with the English language. 


\section{Disclosure of interest}

The authors report no conflict of interest

\section{Funding}

This work was supported by the Ramón y Cajal Grant held by FBO (RYC-201109011). JM-G is supported by the Spanish Ministry of Education, Culture and Sport (FPU 14/06837). CC-S is supported by a grant from the Spanish Ministry of Economy and Competitiveness (BES-2014-068829). FBO is supported by grants from the Spanish Ministry of Science and Innovation (RYC- 2011-09011). The PREFIT Cuenca group was funded by Health and Social Research Center from the University of Castilla-La Mancha. Additional funding was obtained from the University of Granada, Plan Propio de Investigación 2016, Excellence actions: Units of Excellence; Unit of Excellence on Exercise and Health (UCEES). In addition, funding was provided by the SAMID III network, RETICS, the PN I + D + I 2017-2021 (Spain), ISClll-Sub-Directorate General for Research Assessment and Promotion, the European Regional Development Fund (ERDF) (Ref. RD16/0022) and the EXERNET Research Network on Exercise and Health in Special Populations (DEP2005-00046/ACTI). This study is part of Ignacio Merino de Haro's PhD thesis, from the Nutrition and Food Sciences Doctorate Program of the University of Granada. This study is part of Ignacio Merino de Haro's PhD thesis from the Nutrition and Food Sciences Doctorate Program of the University of Granada.

\section{ORCID}

Alejandro Sánchez-Delgado (D) http://orcid.org/0000-0003-1826-1463 Germán Vicente-Rodríguez (D) http://orcid.org/0000-0002-4303-4097

\section{References}

Artero, E. G., Ruiz, J. R., Ortega, F. B., España-Romero, V., Vicente-Rodríguez, G., \& Molnar, D., \& HELENA Study Group. (2011). Muscular and cardiorespiratory fitness are independently associated with metabolic risk in adolescents: The HELENA study. Pediatric Diabetes, 12(8), 704-712.

Bammann, K., Gwozdz, W., Lanfer, A., Barba, G., De Henauw, S., Eiben, G., ... Pigeot, I. (2013). Socioeconomic factors and childhood overweight in Europe: Results from the multi-centre IDEFICS study. Pediatric Obesity, 8(1), 1-12.

Barnett, L., Hinkley, T., Okely, A. D., \& Salmon, J. (2013). Child, family and environmental correlates of children's motor skill proficiency. Journal of Science and Medicine in Sport, 16, 332-336.

Baughcum, A. E., Chamberlin, L. A., Deeks, C. M., Powers, S. W., \& Whitaker, R. C. (2000). Maternal perceptions of overweight preschool children. Pediatrics, 106(6), 1380-1386.

Bray, M. S., Hagberg, J. M., Pérusse, L., Rankinen, T., Roth, S. M., Wolfarth, B., \& Bouchard, C. (2009). The human gene map for performance and health-related fitness phenotypes: The 2006-2007 update. Medicine and Science in Sports and Exercise, 41(1), 35-73.

Bürgi, F., Meyer, U., Niederer, I., Ebenegger, V., Marques-Vidal, P., Granacher, U., ... Puder, J. J. (2010). Socio-cultural determinants of adiposity and physical activity in preschool children: A cross-sectional study. BMC Public Health, 10, 733.

Cadenas-Sánchez, C., Alcántara-Moral, F., Sánchez-Delgado, G., Mora-Gonz ález, J., Martínez-Téllez, B., Herrador-Colmenero, M., ... Ortega, F. B. (2014). Assessment of cardiorespiratory fitness in preschool children: Adaptation of the 20 metres shuttle run test. Nutrición Hospitalaria, 30 (6), 1333-1343.

Cadenas-Sanchez, C., Intemann, T., Labayen, I., Peinado, A. B., Vidal-Conti, J., Sanchis-Moysi, J., ... Ortega, F. B. (2018). Physical fitness reference standards for preschool children: The PREFIT project. Journal of Science and Medicine in Sport. doi:10.1016/j.jsams.2018.09.227

Cadenas-Sanchez, C., Martinez-Tellez, B., Sanchez-Delgado, G., MoraGonzalez, J., Castro-Piñero, J., Löf, M., ... Ortega, F. B. (2016). Assessing physical fitness in preschool children: Feasibility, reliability and practical recommendations for the PREFIT battery. Journal of Science and Medicine in Sport/Sports Medicine Australia, 19(11), 910-915.
Caspersen, C. J., Powell, K. E., \& Christenson, G. M. (1985). Physical activity, exercise, and physical fitness: Definitions and distinctions for health-related research. Public Health Reports, 100(2), 126-131.

Cole, T. J., \& Lobstein, T. (2012). Extended international (IOTF) body mass index cut-offs for thinness, overweight and obesity. Pediatric Obesity, 7 (4), 284-294.

Cutting, T. M., Fisher, J. O., Grimm-Thomas, K., \& Birch, L. L. (1999). Like mother, like daughter: Familial patterns of overweight are mediated by mothers' dietary disinhibition. The American Journal of Clinical Nutrition, 69(4), 608-613.

Drewnowski, A., \& Specter, S. E. (2004). Poverty and obesity: The role of energy density and energy costs. The American Journal of Clinical Nutrition, 79(1), 6-16.

Freitas, D., Maia, J., Beunen, G., Claessens, A., Thomis, M., Marques, A., ... Lefevre, J. (2007). Socio-economic status, growth, physical activity and fitness: The Madeira growth study. Annals of Human Biology, 34(1), 107-122.

GBD 2015 Obesity Collaborators. (2017). Health effects of overweight and obesity in 195 countries over 25 years. The New England Journal of Medicine, 377(1), 13-27.

Hesketh, K., Crawford, D., Salmon, J., Jackson, M., \& Campbell, K. (2007). Associations between family circumstance and weight status of Australian children. International Journal of Pediatric Obesity, 2(2), 86-96.

Huppertz, C., Bartels, M., de Geus, E. J. C., van Beijsterveldt, C. E. M., Rose, R. J., Kaprio, J., \& Silventoinen, K. (2017). The effects of parental education on exercise behavior in childhood and youth: A study in Dutch and Finnish twins. Scandinavian Journal of Medicine \& Science in Sports, 27(10), 1143-1156.

Imhof, K., Faude, O., Donath, L., Bean-Eisenhut, S., Hanssen, H., \& Zahner, L. (2016). The association of socio-economic factors with physical fitness and activity behaviours, spinal posture and retinal vessel parameters in first graders in urban Switzerland. Journal of Sports Sciences, 34(13), 1271-1280.

International Labour Office. (2012). International standard classification of occupations - ISCO-08: Structure, group definitions and correspondence tables. Geneva: International Labour Organization.

Jiménez Pavón, D., Ortega, F. P., Ruiz, J. R., España Romero, V., García Artero, E., Moliner Urdiales, D., \& HELENA Study Group. (2010). Socioeconomic status influences physical fitness in European adolescents independently of body fat and physical activity: The HELENA study. Nutricio'n Hospitalaria, 25(2), 311-316.

Kitsantas, P., \& Gaffney, K. F. (2010). Risk profiles for overweight/obesity among preschoolers. Early Human Development, 86(9), 563-568.

Kobzová, J., Vignerová, J., Bláha, P., Krejcovský, L., \& Riedlová, J. (2004). The 6th nationwide anthropological survey of children and adolescents in the Czech Republic in 2001. Central European Journal of Public Health, 12(3), 126-130.

Lämmle, L., Worth, A., \& Bös, K. (2012). Socio-demographic correlates of physical activity and physical fitness in German children and adolescents. The European Journal of Public Health, 22(6), 880-884.

Lavie, C. J., McAuley, P. A., Church, T. S., Milani, R. V., \& Blair, S. N. (2014), Obesity and cardiovascular diseases: Implications regarding fitness, fatness, and severity in the obesity paradox. Journal of the American College of Cardiology, 63(14), 1345-1354.

Léger, L. A., Mercier, D., Gadoury, C., \& Lambert, J. (1988). The multistage 20 metre shuttle run test for aerobic fitness. Journal of Sports Sciences, 6(2), 93-101.

Lobstein, T., \& Frelut, M.-L. (2003). Prevalence of overweight among children in Europe. Obesity Reviews, 4(4), 195-200.

Mora-Gonzalez, J., Cadenas-Sanchez, C., Martinez-Tellez, B., SanchezDelgado, G., Ruiz, J. R., Léger, L., \& Ortega, F. B. (2017). Estimating VO2max in children aged 5-6 years through the preschool-adapted 20-m shuttle-run test (PREFIT). European Journal of Applied Physiology, 117(11), 2295-2307.

Morley, D., Till, K., Ogilvie, P., \& Turner, G. (2015). Influences of gender and socioeconomic status on the motor proficiency of children in the UK. Human Movement Science, 44, 150-156.

Nakagawa, S., \& Cuthill, I. C. (2007). Effect size, confidence interval and statistical significance: A practical guide for biologists. Biological Reviews, 82(4), 591-605. 
Nightingale, C. M., Rudnicka, A. R., Owen, C. G., Cook, D. G., \& Whincup, P. H. (2011). Patterns of body size and adiposity among UK children of South Asian, black African-Caribbean and white European origin: Child Heart And health Study in England (CHASE study). International Journal of Epidemiology, 40(1), 33-44.

Ortega, F. B., Cadenas-Sánchez, C., Sánchez-Delgado, G., Mora-González, J., Martínez-Téllez, B., Artero, E. G., ... Ruiz, J. R. (2015). Systematic review and proposal of a field-based physical fitness-test battery in preschool children: The PREFIT battery. Sports Medicine (Auckland, N.Z.), 45(4), 533-555.

Ortega, F. B., Lavie, C. J., \& Blair, S. N. (2016). Obesity and cardiovascular disease. Circulation Research, 118(11), 1752-1770.

Ortega, F. B., Ruiz, J. R., Castillo, M. J., \& Sjöström, M. (2008). Physical fitness in childhood and adolescence: A powerful marker of health. International Journal of Obesity (2005), 32(1), 1-11.

Ortega, F. B., Ruiz, J. R., Labayen, I., Hurtig-Wennlöf, A., Harro, J., Kwak, L., ... Sjöström, M. (2013). Role of socio-cultural factors on changes in fitness and adiposity in youth: A 6-year follow-up study. Nutrition, Metabolism and Cardiovascular Diseases, 23(9), 883-890.

Ortega, F. B., Ruiz, J. R., Labayen, I., Lavie, C. J., \& Blair, S. N. (2017). The Fat but Fit paradox: What we know and don't know about it. British Journal of Sports Medicine, 52(3), 151-153.

Ortega, F. B., Silventoinen, K., Tynelius, P., \& Rasmussen, F. (2012). Muscular strength in male adolescents and premature death: Cohort study of one million participants. British Medical Journal, 345, e7279.

Otero, J., Cohen, D. D., Herrera, V. M., Camacho, P. A., Bernal, O., \& LópezJaramillo, P. (2016). Sociodemographic factors related to handgrip strength in children and adolescents in a middle income country: The SALUS study. American Journal of Human Biology, 29, 1.

Ruiz, J. R., Castro-Piñero, J., Artero, E. G., Ortega, F. B., Sjöström, M., Suni, J., \& Castillo, M. J. (2009). Predictive validity of health-related fitness in youth: A systematic review. British Journal of Sports Medicine, 43(12), 909-923.

Sanchez-Delgado, G., Cadenas-Sanchez, C., Mora-Gonzalez, J., Martinez-Tellez, B., Chillón, P., Löf, M., ... Ruiz, J. R. (2015). Assessment of handgrip strength in preschool children aged 3 to 5 years. The Journal of Hand Surgery, European Volume, 40(9), 966-972.
Sandercock, G. R. H., Lobelo, F., Correa-Bautista, J. E., Tovar, G., Cohen, D. D., Knies, G., \& Ramírez-Vélez, R. (2017). The relationship between socioeconomic status, family income, and measures of muscular and cardiorespiratory fitness in Colombian schoolchildren. The Journal of Pediatrics, 185, 81-87.

Schmutz, E. A., Leeger-Aschmann, C. S., Radtke, T., Muff, S., Kakebeeke, T. H., Zysset, A. E., ... Kriemler, S. (2017). Correlates of preschool children's objectively measured physical activity and sedentary behavior: A crosssectional analysis of the SPLASHY study. International Journal of Behavioral Nutrition and Physical Activity, 14(1), 1.

Shrewsbury, V., \& Wardle, J. (2008). Socioeconomic status and adiposity in childhood: A systematic review of cross-sectional studies 1990-2005. Obesity, 16(2), 275-284.

Steene-Johannessen, J., Anderssen, S. A., Kolle, E., \& Andersen, L. B. (2009). Low muscle fitness is associated with metabolic risk in youth. Metabolic Risk in Youth. Med. Sci. Sports Exerc, 41(7), 1361-1367.

Trost, S. G., \& Loprinzi, P. D. (2011). Parental influences on physical activity behavior in children and adolescents: A brief review. American Journal of Lifestyle Medicine, 5(2), 171-181.

Wang, C., \& Sweetman, A. (2013). Gender, family status and physician labour supply. Social Science \& Medicine, 94, 17-25.

Winkleby, M. A., Kraemer, H. C., Ahn, D. K., \& Varady, A. N. (1998). Ethnic and socioeconomic differences in cardiovascular disease risk factors: Findings for women from the third national health and nutrition examination survey, 1988-1994. JAMA, 280(4), 356-362.

World Health Organization (WHO). (2015). World Health Organization. Geneva: Global Strategy on Diet, Physical Activity and Health.

Wu, S., Ding, Y., Wu, F., Li, R., Hu, Y., Hou, J., \& Mao, P. (2015). Socioeconomic position as an intervention against overweight and obesity in children: A systematic review and meta-analysis. Scientific Reports, 5, 11354.

Yannakoulia, M., Papanikolaou, K., Hatzopoulou, I., Efstathiou, E., Papoutsakis, C., \& Dedoussis, G. V. (2008). Association between family divorce and children's BMI and meal patterns: The GENDAl study. Obesity, 16(6), 1382-1387. 